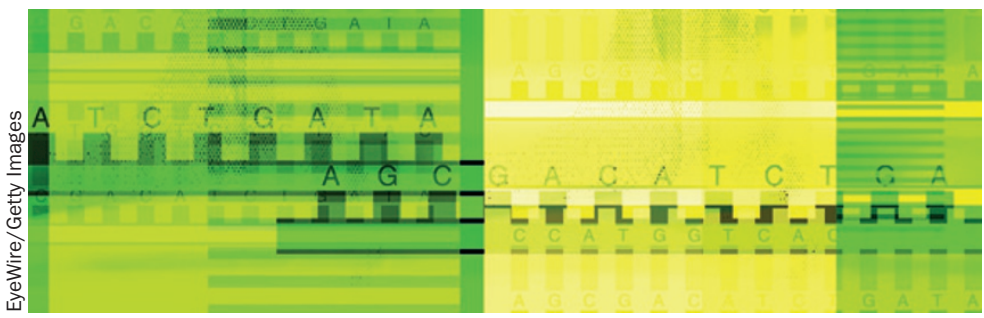

\title{
Glioblastoma landscape revealed
}

Genetic changes that occur in glioblastoma multiforme (GBM) tumours have been previously identified, but the functional consequence of many of these mutations is yet to be established. Against this background, researchers led by Anna Lasorella, Raul Rabadan and Antonio Iavarone used a new statistical approach to identify potential driver mutations. As the researchers pointed out "we anticipated that some of the driver mutations might be important tumour-addicting events, therefore, providing new therapeutic targets for personalized therapy of GBM."

GBM affects over 10,000 patients in the USA each year, and is associated with a short median survival of only $12-15$ months. It is also a complex disease in terms of genetic heterogeneity, with an abundance of passenger mutations and copy-number variations that have made the identification of the true drivers of this disease challenging. The present study used a combination of techniques to overcome this obstacle; as the researchers put it "we used in-depth computational and experimental methodologies. The first infers potential driver mutations and segregates them from the many passenger mutations. The second validates experimentally, often in patient-derived glioma stem cells, the functional significance of the most important new driver mutations and gene fusions and the potential value as therapeutic targets in vitro and in vivo."

An initial assessment of 469 GBM samples generated a list of 67 genes that were defined as recurrently mutated without copy-number alterations, in regions of focal and recurrent amplifications, or in regions of focal and recurrent deletions. These 67 genes included nearly all the genes that had previously been implicated as having a role in GBM. The screen also identified 52 new candidate driver genes. From this pool of genes, 24 were selected and resequenced in an independent group of 83 glioblastoma samples -18 genes were somatically mutated in this panel.

From these 18 new GBM genes, LZTR1 (encoding an adaptor of CUL3containing E3 ligase complexes) was identified to be concurrently targeted in GBM by mutations and copy-number loss. This feature makes it a prime candidate for a tumour-suppressor gene. Cell biology experiments support this function, and indicate that LZTR1 inactivation in GBM is a driver of self-renewal and the growth of glioma spheres. Another topranking gene was CTNND2 (encoding $\delta$-catenin), inactivation of which was shown to be a key driver of the aggressive mesenchymal phenotype of GBM.

In addition to these genes, three new receptor-tyrosine kinase (RTK) genes (FGFR, EGFR and NTRK1) were found to harbour recurrent gene fusions. The EGFR fusion is of particular clinical interest and the researchers "plan to use the new information to target in GBM patients the gene fusions implicating RTK-coding that we have identified with drugs already available in the clinic."

Rebecca Kirk 